\title{
Optimization of a two-way MIMO amplify-and-forward relay network
}

\author{
Ying Zhang ${ }^{1 *}$, Yinjiang Chen ${ }^{1}$, Chuanyi Pan ${ }^{1}$, Huapeng Zhao ${ }^{2}$ and Ning Kang ${ }^{1}$
}

\begin{abstract}
In this paper, we consider optimization of a two-way multiple-input multiple-output (MIMO) amplify-and-forward relay network which consists of a pair of transceivers and several relay nodes. Multiple antennas are equipped on the transceivers and relays. Multiple access broadcast scheme which finishes communication in two time slots is considered. In the first time slot, signals received by the relays are scaled by several beamforming matrices. In the second time slot, the relays transmit the scaled signals to the two transceivers. Upon receiving these signals, a MIMO equalizer is implemented at each transceiver to recover the desired signal. In this paper, zero forcing equalizers are used. Joint optimization of the beamforming matrices and the equalizers are realized using the following criteria: 1) the total relay transmission power is minimized subject to the minimal output signal-to-noise ratio (SNR) constraint at each transceiver, 2) the minimal output SNR of the two transceivers is maximized subject to total relay transmission power constraint, and 3) the minimal output SNR of the two transceivers is maximized subject to individual relay transmission power constraint. It is shown that the proposed optimization problems can be formulated as the second-order cone programming problems which can be solved efficiently. The validity of the proposed algorithm is verified by computer simulations.
\end{abstract}

Keywords: Amplify-and-forward; Beamforming; Multiple-input multiple-output; Relaying; Second-order cone programming; Zero-forcing

\section{Introduction}

Relaying technique is capable of extending communication range and coverage by providing link to shadowed users via relay nodes, and it received extensive study in recent years. For collaborative relaying technique, the network with a single pair of users and multiple relay nodes equipped with single antenna has been widely investigated [1-7]. Zheng [1] assumed perfect knowledge of channel-state information (CSI) and proposed to optimize the beamforming vector by maximizing the destination signal-to-noise ratio (SNR) subject to total and local relay transmission power constraints. In [2], similar optimization criterion was used in the case that only the second-order statistics of CSI are available. In $[3,4]$, quantized CSI was considered. Quantizer at each relay and beamforming vectors at destination were optimized to minimize the uncoded bit error rate. In [5],

\footnotetext{
*Correspondence: zh0045ng@e.ntu.edu.sg

${ }^{1}$ College of Electronic Engineering, University of Electronic Science and Technology of China, Xiyuan Avenue, 610066 Chengdu, China

Full list of author information is available at the end of the article
}

the minimizing mean square error (MMSE) criterion was adopted to optimize the beamforming coefficients. The advantage of this algorithm lies in its ability to adaptively allocate transmission power of each relay. In recent, a so-called filter-and-forward distributed beamforming technique was proposed in [6]. Different from previous algorithms, this method solved the problem of relay beamforming in frequency selective environments, where a finite impulse response filter is used at each relay. Apart from the above mentioned one-way scheme, a twoway relaying strategy was proposed in [7]. In a two-way relaying scheme, the relays cooperate with each other to establish the connection between two transceivers. The design of the beamformer should simultaneously satisfy the requirements from the two transceivers. In [8], an optimization strategy was proposed to optimize the performance of a two-way single-input single-output relaying network.

Multiple relay nodes create a virtual multiple-input multiple-output (MIMO) environment at the relay layer.

\section{望 Springer}

C 2014 Zhang et al: licensee Springer. This is an Open Access article distributed under the terms of the Creative Commons Attribution License (http://creativecommons.org/licenses/by/4.0), which permits unrestricted use, distribution, and reproduction in any medium, provided the original work is properly credited. 
With multiple antennas equipped at transmitting and receiving nodes, user nodes can also employ advantages of MIMO techniques, such as spatial multiplexing, spacetime coding, and beamforming. Attracted by these merits, more and more algorithms are proposed to optimize MIMO relay networks [9-14] and virtual MIMO relay networks [15-18]. Most of these algorithms consider oneway communication scheme, where various optimization criteria are adopted, such as maximizing the destination SNR and minimizing the total system/relay transmission power, MMSE, ZF (zero force), etc. For a single pair of users and multiple relays, a unified algorithm which computes the optimal linear transceivers jointly at the source node and the relay nodes for two-way amplifyand-forward (AF) protocols was proposed [14]. The optimization algorithm was designed based on maximization of sum rate and MMSE. In [16], a two-way scheme was considered for a relay network with multiple users and single relay. The network was optimized using MMSE criterion at the destination subject to power constraint on relays.

In this paper, we consider a two-way MIMO relay network with one pair of transceivers and multiple relays, where the two transceivers are each equipped with $M$ antennas, and every relay is equipped with $N$ antennas. We assume that relays receive the mixture of signals from two transceivers in the first time slot. With perfect CSI, relays scale the received signals and then transmit these signals to the transceivers in the second time slot. Finally, a MIMO equalizer is used at each transceiver to recover the desired signal. To achieve power-efficient communication, beamforming coefficient matrices are optimized based on three criteria which are designed based on the minimal output SNR and relay transmission power. Meanwhile, the MIMO equalizer is optimized by imposing the ZF constraint. It is shown that the proposed optimization problems can be formulated as the second-order cone programming (SOCP) problems, which can be efficiently solved using the cvx toolbox [19]. Contributions of this paper are summarized as follows. First, two-way communication scheme of a MIMO relay network consists of multiple relays with multiple antennas is firstly considered. Second,the proposed optimization problem is formulated as an SOCP problem which can be solved efficiently.

The rest of this paper is organized as follows. The 'Problem formulation' section presents the problem formulation. The 'Mathematical approximation' section develops mathematical preparation for optimizing the considered relay network, and 'Optimization of the proposed relay network' section gives a detailed optimization procedure to derive the optimal beamforming and equalization matrices. In the 'Computer simulations' section, computer simulations are conducted to demon- strate validity of the proposed algorithm. Finally, conclusions and discussions are presented in the 'Conclusions' section.

\section{Notation}

Bold lower case is used for vectors, while bold capital letters for matrices. I denotes the unit matrix, $*$ denotes the complex conjugate operation, $T$ denotes the matrix transposition operation, and $H$ denotes the complex conjugate transposition operation. $\operatorname{tr}(\mathbf{A})$ is the trace of matrix $\mathbf{A}$. $\operatorname{vec}(\mathbf{A})$ stacks the columns of $\mathbf{A}$ into a single column vector. $\otimes$ denotes the Kronecker product, and $\left(\mathbf{A}_{0} \oplus \ldots \oplus \mathbf{A}_{N}\right)$ yields a block diagonal matrix with block elements given by $\mathbf{A}_{i}$.

\section{Problem formulation}

Figure 1 depicts a two-way MIMO relay scheme, where $\mathbf{W}_{i}(i=1,2, \ldots, L)$ is the beamforming matrix for the $i$ th relay and $\mathbf{D}_{j}(j=1,2)$ is the equalizer for the $j$ th transceiver. Transceiver 1 and transceiver 2 are both equipped with $M$ antennas. It is supposed that $L$ relays equipped with $N$ antennas are used. Flat fading channels are considered. We assume that no direct link exists between the two transceivers. The channel matrix from transceiver 1 to the $i$ th relay is denoted as $\mathbf{H}_{i}(i=$ $1,2, \ldots, L)$, and the one from the $i$ th relay to transceiver 2 is denoted as $\mathbf{G}_{i}$, where $\mathbf{H}_{i} \in \mathbb{C}^{N \times M}$ and $\mathbf{G}_{i} \in \mathbb{C}^{M \times N}$ consist of independent complex Gaussian variables. It is assumed that these channels are reciprocal, i.e., the channel matrix from the $i$ th relay to transceiver 1 is $\mathbf{H}_{i}^{T}$, and the one from transceiver 2 to the $i$ th relay is $\mathbf{G}_{i}^{T}$. In the first time slot, each transceiver sends messages to the $L$ relays. With the knowledge of $\mathbf{H}_{i}$ and $\mathbf{G}_{i}$ (which can be obtained via training), $\mathbf{W}_{i}$ is computed. In the second time slot, the relays scale the received signals according to $\mathbf{W}_{i}$ and then transmit these signals to the two transceivers. After receiving signals, a MIMO equalizer denoted as $\mathbf{D}_{j}, j=1,2$ is used at each transceiver. The aim of this paper is to optimize the performance of the relay network by designing $\mathbf{W}_{i}$ and $\mathbf{D}_{j}$.

The mixture of signals received by the $i$ th relay can be expressed as

$$
\mathbf{r}_{i}=\mathbf{H}_{i} \mathbf{s}_{1}+\mathbf{G}_{i}^{T} \mathbf{s}_{2}+\mathbf{v}_{i}
$$

where $\mathbf{s}_{1}$ and $\mathbf{s}_{2}$ are transmitted signals with covariance matrices $P_{1} \mathbf{I}$ and $P_{2} \mathbf{I}$, respectively, and they are independent from each other. $\mathbf{v}_{i}$ denotes the additive Gaussian noise (AGN) with covariance matrix $\mathbf{R}_{v i}$ at the $i$ th relay. In this paper, it is assumed that AGN is white, and its covariance matrix is identical for all relays, i.e., $\mathbf{R}_{v i}=\sigma_{v}^{2} \mathbf{I}, \forall i=$ $1, \ldots, L$. 


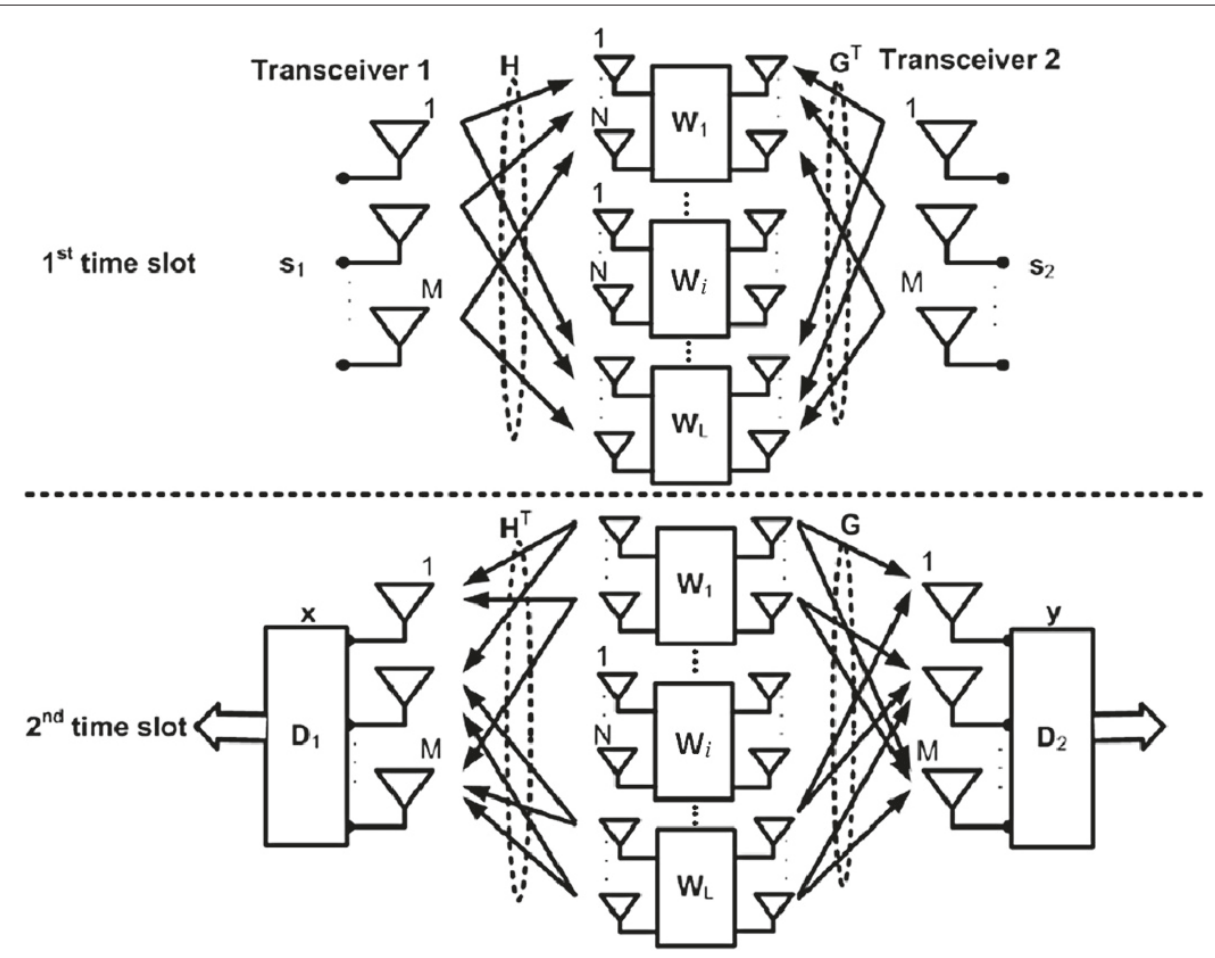

Figure $1 \mathrm{~A}$ two-way MIMO relay scheme.

In the second time slot, transceiver 1 and transceiver 2 each receives

$$
\begin{aligned}
\mathbf{x}= & \sum_{i=1}^{L} \mathbf{H}_{i}^{T} \mathbf{W}_{i} \mathbf{r}_{i}+\mathbf{v}_{x} \\
= & \sum_{i=1}^{L} \mathbf{H}_{i}^{T} \mathbf{W}_{i} \mathbf{H}_{i} \mathbf{s}_{1}+\sum_{i=1}^{L} \mathbf{H}_{i}^{T} \mathbf{W}_{i} \mathbf{G}_{i}^{T} \mathbf{s}_{2} \\
& +\sum_{i=1}^{L} \mathbf{H}_{i}^{T} \mathbf{W}_{i} \mathbf{v}_{i}+\mathbf{v}_{x} \\
\mathbf{y}= & \sum_{i=1}^{L} \mathbf{G}_{i} \mathbf{W}_{i} \mathbf{r}_{i}+\mathbf{v}_{y} \\
= & \sum_{i=1}^{L} \mathbf{G}_{i} \mathbf{W}_{i} \mathbf{H}_{i} \mathbf{s}_{1}+\sum_{i=1}^{L} \mathbf{G}_{i} \mathbf{W}_{i} \mathbf{G}_{i}^{T} \mathbf{s}_{2} \\
& +\sum_{i=1}^{L} \mathbf{G}_{i} \mathbf{W}_{i} \mathbf{v}_{i}+\mathbf{v}_{y},
\end{aligned}
$$

where $\mathbf{v}_{x}$ and $\mathbf{v}_{y}$ denote AGN at transceiver 1 and transceiver 2, and their covariance matrices are assumed to be $\sigma_{x}^{2} \mathbf{I}$ and $\sigma_{y}^{2} \mathbf{I}$, respectively. $\mathbf{s}_{1}$ and $\mathbf{s}_{2}$ are known by transceiver 1 and transceiver 2 , respectively. If $\mathbf{G}_{i}, \mathbf{H}_{i}$, and $\mathbf{W}_{i}$ are available to transceivers, terms containing $\mathbf{s}_{1}$ and $\mathbf{s}_{2}$ can be subtracted from Equations $2 \mathrm{a}$ and $2 \mathrm{~b}$, respectively. Equations $2 \mathrm{a}$ and $2 \mathrm{~b}$ are then rewritten as

$$
\begin{aligned}
& \overline{\mathbf{x}}=\sum_{i=1}^{L} \mathbf{H}_{i}^{T} \mathbf{W}_{i} \mathbf{G}_{i}^{T} \mathbf{s}_{2}+\sum_{i=1}^{L} \mathbf{H}_{i}^{T} \mathbf{W}_{i} \mathbf{v}_{i}+\mathbf{v}_{x}, \\
& \overline{\mathbf{y}}=\sum_{i=1}^{L} \mathbf{G}_{i} \mathbf{W}_{i} \mathbf{H}_{i} \mathbf{s}_{1}+\sum_{i=1}^{L} \mathbf{G}_{i} \mathbf{W}_{i} \mathbf{v}_{i}+\mathbf{v}_{y} .
\end{aligned}
$$

A MIMO equalizer is used at each transceiver; thereby, the restored signal after equalization is expressed as

$$
\begin{aligned}
& \widehat{\mathbf{s}}_{2}=\mathbf{D}_{1} \overline{\mathbf{x}}, \\
& \widehat{\mathbf{s}}_{1}=\mathbf{D}_{2} \overline{\mathbf{y}} .
\end{aligned}
$$

Based on Equations 1 to 4, the total relay transmission power and terminal SNRs are defined as follows:

1) Total relay transmission power:

$$
\begin{aligned}
P_{\mathrm{r}}= & \sum_{i=1}^{L} E\left[\left\|\mathbf{W}_{i} \mathbf{r}_{i}\right\|_{2}^{2}\right] \\
= & \sum_{i=1}^{L}\left[P_{1} \operatorname{tr}\left(\mathbf{W}_{i} \mathbf{H}_{i} \mathbf{H}_{i}^{H} \mathbf{W}_{i}^{H}\right)\right. \\
& \left.+P_{2} \operatorname{tr}\left(\mathbf{W}_{i} \mathbf{G}_{i}^{T} \mathbf{G}_{i}^{*} \mathbf{W}_{i}^{H}\right)+\sigma_{v}^{2} \operatorname{tr}\left(\mathbf{W}_{i} \mathbf{W}_{i}^{H}\right)\right]
\end{aligned}
$$


2) Terminal SNR at transceiver 1 :

$$
\begin{aligned}
\mathrm{SNR}_{1} & =\frac{E\left[\left\|\mathbf{D}_{1} \sum_{i=1}^{L} \mathbf{H}_{i}^{T} \mathbf{W}_{i} \mathbf{G}_{i}^{T} \mathbf{s}_{2}\right\|_{2}^{2}\right]}{E\left[\left\|\mathbf{D}_{1} \sum_{i=1}^{L} \mathbf{H}_{i}^{T} \mathbf{W}_{i} \mathbf{v}_{i}+\mathbf{D}_{1} \mathbf{v}_{x}\right\|_{2}^{2}\right]} \\
= & \frac{P_{2} \operatorname{tr}\left(\mathbf{D}_{1}\left(\sum_{i=1}^{L} \mathbf{H}_{i}^{T} \mathbf{W}_{i} \mathbf{G}_{i}^{T}\right)\left(\sum_{i=1}^{L} \mathbf{G}_{i}^{*} \mathbf{W}_{i}^{H} \mathbf{H}_{i}^{*}\right) \mathbf{D}_{1}^{H}\right)}{\operatorname{tr}\left(\mathbf{D}_{1}\left(\sigma_{v}^{2} \sum_{i=1}^{L} \mathbf{H}_{i}^{T} \mathbf{W}_{i} \sum_{i=1}^{L} \mathbf{W}_{i}^{H} \mathbf{H}_{i}^{*}+\sigma_{x}^{2} \mathbf{I}\right) \mathbf{D}_{1}^{H}\right)}
\end{aligned}
$$

3) Terminal SNR at transceiver 2:

$$
\begin{aligned}
\mathrm{SNR}_{2}= & \frac{E\left[\left\|\mathbf{D}_{2} \sum_{i=1}^{L} \mathbf{G}_{i} \mathbf{W}_{i} \mathbf{H}_{i} \mathbf{s}_{1}\right\|_{2}^{2}\right]}{E\left[\left\|\mathbf{D}_{2} \sum_{i=1}^{L} \mathbf{G}_{i} \mathbf{W}_{i} \mathbf{v}_{i}+\mathbf{D}_{2} \mathbf{v}_{y}\right\|_{2}^{2}\right]} \\
= & \frac{P_{1} \operatorname{tr}\left(\mathbf{D}_{2}\left(\sum_{i=1}^{L} \mathbf{G}_{i} \mathbf{W}_{i} \mathbf{H}_{i}\right)\left(\sum_{i=1}^{L} \mathbf{H}_{i}^{H} \mathbf{W}_{i}^{H} \mathbf{G}_{i}^{H}\right) \mathbf{D}_{2}^{H}\right)}{\operatorname{tr}\left(\mathbf{D}_{2}\left(\sigma_{v}^{2} \sum_{i=1}^{L} \mathbf{G}_{i} \mathbf{W}_{i} \sum_{i=1}^{L} \mathbf{W}_{i}^{H} \mathbf{G}_{i}^{H}+\sigma_{y}^{2} \mathbf{I}\right) \mathbf{D}_{2}^{H}\right)} .
\end{aligned}
$$

In subsequent sections, $P_{\mathrm{r}}, \mathrm{SNR}_{1}$, and $\mathrm{SNR}_{2}$ will be used to optimize beamforming matrices and MIMO equalizers.

\section{Mathematical approximation}

From (5) to (7), it seems difficult to directly evaluate $\mathbf{W}_{i}$ and $\mathbf{D}_{j}$ because they appear in both signal and noise terms. Therefore, before designing beamformers, three lemmas are derived to make $\mathbf{W}_{i}$ and $\mathbf{D}_{j}$ solvable.

Lemma 1. Total relay transmission power can be expressed as a quadratic function of $\mathbf{w}$ :

$$
P_{\mathrm{r}}=P_{1} \mathbf{w}^{H} \overline{\mathbf{H}} \mathbf{w}+P_{2} \mathbf{w}^{H} \mathbf{G} \mathbf{w}+\sigma_{v}^{2} \mathbf{w}^{H} \mathbf{w},
$$

where

$$
\begin{aligned}
\mathbf{w}_{i} & =\operatorname{vec}\left(\mathbf{W}_{i}\right), i=1, \ldots, L, \\
\mathbf{w} & =\left(\mathbf{w}_{1}^{T}, \ldots, \mathbf{w}_{L}^{T}\right)^{T}, \\
\overline{\mathbf{H}}_{i k} & =\left(\mathbf{h}_{i k}^{T} \otimes \mathbf{I}_{N}\right), \\
\overline{\mathbf{H}} & =\sum_{k=1}^{M} \overline{\mathbf{H}}_{1 k}^{H} \overline{\mathbf{H}}_{1 k} \oplus \ldots \oplus \sum_{k=1}^{M} \overline{\mathbf{H}}_{L k}^{H} \overline{\mathbf{H}}_{L k}, \\
\mathbf{G}_{i k} & =\left(\overline{\mathbf{g}}_{i k}^{T} \otimes \mathbf{I}_{N}\right), \\
\mathbf{G} & =\sum_{k=1}^{M} \mathbf{G}_{1 k}^{H} \mathbf{G}_{1 k} \oplus \ldots \oplus \sum_{k=1}^{M} \mathbf{G}_{L k}^{H} \mathbf{G}_{L k},
\end{aligned}
$$

are defined. The notation of $\mathbf{h}_{i k}$ and $\overline{\mathbf{g}}_{i k}$ is given in the proof.
Proof. From (5), the $k$ th column of $\left(\mathbf{W}_{i} \mathbf{H}_{i}\right)$ can be expressed as $\left(\mathbf{h}_{i k}^{T} \otimes \mathbf{I}_{N}\right) \mathbf{w}_{i}$, where $\mathbf{h}_{i k}$ denotes the $k$ th column of matrix $\mathbf{H}_{i}$. Therefore, the trace of $\left(\mathbf{W}_{i} \mathbf{H}_{i} \mathbf{H}_{i}^{H} \mathbf{W}_{i}^{H}\right)$ is given by the Frobenious norm of $\left(\mathbf{W}_{i} \mathbf{H}_{i}\right)$, which can be computed as

$\operatorname{tr}\left(\mathbf{W}_{i} \mathbf{H}_{i} \mathbf{H}_{i}^{H} \mathbf{W}_{i}^{H}\right)=\mathbf{w}_{i}^{H} \sum_{k=1}^{M}\left(\mathbf{h}_{i k}^{T} \otimes \mathbf{I}_{N}\right)^{H}\left(\mathbf{h}_{i k}^{T} \otimes \mathbf{I}_{N}\right) \mathbf{w}_{i}$.

Expressing the trace of $\left(\mathbf{W}_{i} \mathbf{G}_{i}^{T} \mathbf{G}_{i}^{*} \mathbf{W}_{i}^{H}\right)$ in the similar way of (9) and substituting it and (9) into (5) yield

$$
\begin{aligned}
P_{\mathrm{r}}= & P_{1} \sum_{i=1}^{L} \mathbf{w}_{i}^{H} \sum_{k=1}^{M}\left(\mathbf{h}_{i k}^{T} \otimes \mathbf{I}_{N}\right)^{H}\left(\mathbf{h}_{i k}^{T} \otimes \mathbf{I}_{N}\right) \mathbf{w}_{i} \\
& +P_{2} \sum_{i=1}^{L} \mathbf{w}_{i}^{H} \sum_{k=1}^{M}\left(\overline{\mathbf{g}}_{i k}^{T} \otimes \mathbf{I}_{N}\right)^{H}\left(\overline{\mathbf{g}}_{i k}^{T} \otimes \mathbf{I}_{N}\right) \mathbf{w}_{i} \\
& +\sigma_{v}^{2} \sum_{i=1}^{L} \mathbf{w}_{i}^{H} \mathbf{w}_{i},
\end{aligned}
$$

where $\overline{\mathbf{g}}_{i k}$ denotes the $k$ th column of matrix $\mathbf{G}_{i}^{T}$.

Using the definitions in Lemma 1, (8) can be derived.

SNR constraint is usually used in optimizing a relay network. For our problem, constraints on destination SNRs are expressed as

$$
\begin{aligned}
& \mathrm{SNR}_{1} \geq \gamma_{1}, \\
& \mathrm{SNR}_{2} \geq \gamma_{2},
\end{aligned}
$$

where $\gamma_{1}$ and $\gamma_{2}$ are required SNRs at transceiver 1 and transceiver 2, respectively. From (6) and (7), it is seen that (10) is related to $\mathbf{W}_{i}$ and $\mathbf{D}_{j}$ in a complicated form. In the rest of this section, two lemmas are derived to transform (10) into a manageable form.

The ZF constraint requires that

$$
\begin{aligned}
& \mathbf{D}_{1} \sum_{i=1}^{L} \mathbf{H}_{i}^{T} \mathbf{W}_{i} \mathbf{G}_{i}^{T}=\mathbf{I}, \\
& \mathbf{D}_{2} \sum_{i=1}^{L} \mathbf{G}_{i} \mathbf{W}_{i} \mathbf{H}_{i}=\mathbf{I},
\end{aligned}
$$

where $\mathbf{D}_{1}$ and $\mathbf{D}_{2}$ are defined as the left pseudoinverse of $\sum_{i=1}^{L} \mathbf{H}_{i}^{T} \mathbf{W}_{i} \mathbf{G}_{i}^{T}$ and $\sum_{i=1}^{L} \mathbf{G}_{i} \mathbf{W}_{i} \mathbf{H}_{i}$, respectively. 
Lemma 2. From definitions of $\mathbf{D}_{1}$ and $\mathbf{D}_{2}$ given above, if $\mathbf{D}_{1}^{H} \mathbf{D}_{1}$ and $\mathbf{D}_{2}^{H} \mathbf{D}_{2}$ are diagonal matrices, we have

$$
\begin{aligned}
& \operatorname{tr}\left(\mathbf{D}_{1}^{H} \mathbf{D}_{1}\right)=\sum_{i=1}^{M} \frac{1}{\left|\phi_{i i}\right|^{2} \sigma_{i}}, \\
& \operatorname{tr}\left(\mathbf{D}_{2}^{H} \mathbf{D}_{2}\right)=\sum_{i=1}^{M} \frac{1}{\left|\widetilde{\phi}_{i i}\right|^{2} \widetilde{\sigma}_{i}},
\end{aligned}
$$

where the definitions of $\phi_{i i}, \widetilde{\phi}_{i i}, \sigma_{i}$, and $\widetilde{\sigma}_{i}$ are given in the proof.

Proof. It is straightforward to show that

$$
\mathbf{D}_{1} \mathbf{D}_{1}^{H}=\left(\sum_{i=1}^{L} \mathbf{G}_{i}^{*} \mathbf{W}_{i}^{H} \mathbf{H}_{i}^{*} \sum_{i=1}^{L} \mathbf{H}_{i}^{T} \mathbf{W}_{i} \mathbf{G}_{i}^{T}\right)^{-1} .
$$

If we define $\underline{\mathbf{H}}=\left[\mathbf{H}_{1}^{T}, \ldots, \mathbf{H}_{L}^{T}\right]^{T}, \underline{\mathbf{W}}=\mathbf{W}_{1} \oplus \ldots \mathbf{W}_{L}$, and $\underline{\mathbf{G}}=\left[\mathbf{G}_{1}, \ldots, \mathbf{G}_{L}\right]$, (13) can be expressed as

$$
\mathbf{D}_{1} \mathbf{D}_{1}^{H}=\left(\underline{\mathbf{G}}^{*} \underline{\mathbf{W}}^{H} \underline{\mathbf{H}}^{*} \underline{\mathbf{H}}^{T} \underline{\mathbf{W}} \underline{\mathbf{G}}^{T}\right)^{-1} .
$$

Suppose that the eigendecomposition of $\underline{\mathbf{H}}^{*} \underline{\mathbf{H}}^{T}$ is given by

$$
\begin{aligned}
\underline{\mathbf{H}}^{*} \underline{\mathbf{H}}^{T} & =\left(\begin{array}{ll}
\mathbf{U} & \mathbf{U}_{\|}
\end{array}\right)\left(\begin{array}{ll}
\boldsymbol{\Lambda} & \mathbf{0} \\
\mathbf{0} & \mathbf{0}
\end{array}\right)\left(\begin{array}{l}
\mathbf{U}^{H} \\
\mathbf{U}_{\|}^{H}
\end{array}\right) \\
& =\mathbf{U} \mathbf{\Lambda} \mathbf{U}^{H} .
\end{aligned}
$$

In (15), the diagonal of $\boldsymbol{\Lambda}$ consists of $M$ nonzero eigenvalues. The matrix $\mathbf{U}$ consists of all the eigenvectors corresponding to these nonzero eigenvalues. $\mathbf{U}_{\|}$consists of column vectors which are linearly dependent on columns of $\mathbf{U}$. The dependence of eigenvectors is caused by rank deficiency of $\underline{\mathbf{H}}^{*} \underline{\mathbf{H}}^{T}$ whose effective rank is $M$.

We define $\underline{\overline{\mathbf{W}}}=\underline{\mathbf{W}} \underline{\mathbf{G}}^{T}$, and assume that $\underline{\overline{\mathbf{W}}}$ can be represented by the complete orthogonal basis in the $N L$ dimensional space, where $\mathbf{U}$ is contained in the complete orthogonal basis, i.e.,

$$
\overline{\mathbf{W}}=\left(\begin{array}{ll}
\mathbf{U} \mathbf{U}_{\perp}
\end{array}\right)\left(\begin{array}{c}
\boldsymbol{\Phi} \\
\boldsymbol{\Phi}_{\perp}
\end{array}\right) .
$$

In (16), $\mathbf{U} \in \mathbb{C}^{N L \times M}, \boldsymbol{\Phi} \in \mathbb{C}^{M \times M}$, and $\mathbf{U}_{\perp}$ consist of $N-$ $M$ orthogonal basis of the $N L$-dimensional space, which can be obtained via Gram-Schmidt procedure based on $\mathbf{U}$.

Substituting (15) and (16) into (14) yields

$$
\begin{aligned}
\mathbf{D}_{1} \mathbf{D}_{1}^{H}= & \left(\left(\begin{array}{ll}
\boldsymbol{\Phi}^{H} & \boldsymbol{\Phi}_{\perp}^{H}
\end{array}\right)\left(\begin{array}{c}
\mathbf{U}^{H} \\
\mathbf{U}_{\perp}^{H}
\end{array}\right) \mathbf{U} \boldsymbol{\Lambda} \mathbf{U}^{H}\right. \\
& \left.\left(\begin{array}{l}
\mathbf{U} \\
\mathbf{U}_{\perp}
\end{array}\right)\left(\begin{array}{c}
\boldsymbol{\Phi} \\
\boldsymbol{\Phi}_{\perp}
\end{array}\right)\right)^{-1}=\left(\boldsymbol{\Phi}^{H} \boldsymbol{\Lambda} \boldsymbol{\Phi}\right)^{-1} .
\end{aligned}
$$

From (17), it is seen that

$$
\operatorname{tr} \mid\left(\mathbf{D}_{1}^{H} \mathbf{D}_{1}\right)=\operatorname{tr}\left(\mathbf{D}_{1} \mathbf{D}_{1}^{H}\right) \leq \sum_{i=1}^{M} \frac{1}{\left|\phi_{i i}\right|^{2} \sigma_{i}},
$$

where $\phi_{i i}$ and $\sigma_{i}$ are the $i$ th diagonal element of $\boldsymbol{\Phi}$ and $\boldsymbol{\Lambda}$, respectively.

Similarly, for $\mathbf{D}_{2}$, we have

$$
\operatorname{tr}\left(\mathbf{D}_{2}^{H} \mathbf{D}_{2}\right) \leq \sum_{i=1}^{M} \frac{1}{\left|\widetilde{\phi}_{i i}\right|^{2} \widetilde{\sigma}_{i}}
$$

where $\widetilde{\sigma}_{i}$ is the $i$ th nonzero eigenvalue of $\underline{\mathbf{G}}^{H} \underline{\mathbf{G}} . \widetilde{\phi}_{i i}$ is the $i$ th diagonal element of $\tilde{\boldsymbol{\Phi}}$, where $\underline{\mathbf{W H}}=\left(\begin{array}{ll}\tilde{\mathbf{U}} & \tilde{\mathbf{U}}_{\perp}\end{array}\right)\left(\begin{array}{c}\tilde{\boldsymbol{\Phi}} \\ \tilde{\boldsymbol{\Phi}}_{\perp}\end{array}\right)$, and $\widetilde{\mathbf{U}}$ consists of eigenvectors of $\underline{\mathbf{G}}^{H} \underline{\mathbf{G}}$ corresponding to its nonzero eigenvalues.

Lemma 3. Inequalities (10) can be relaxed as

$$
\begin{aligned}
& \frac{\sigma_{v}^{2} \mathbf{w}^{H} \mathbf{H} \mathbf{w}+M \sigma_{x}^{2}}{\mathbf{w}^{H} \mathbf{d}_{i} \mathbf{d}_{i}^{H} \mathbf{w} \sigma_{i}} \leq \frac{1}{\gamma_{1}}, \forall i=1, \ldots, M, \\
& \frac{\sigma_{v}^{2} \mathbf{w}^{H} \overline{\mathbf{G}} \mathbf{w}+M \sigma_{y}^{2}}{\mathbf{w}^{H} \widetilde{\mathbf{d}}_{i} \widetilde{\mathbf{d}}_{i}^{H} \mathbf{w} \widetilde{\sigma}_{i}} \leq \frac{1}{\gamma_{2}}, \forall i=1, \ldots, M, \\
& \mathbf{w}^{H} \mathbf{e}_{i j}^{*}=0, \forall i=1, \ldots, M, j \neq i, \\
& \mathbf{w}^{H} \widetilde{\mathbf{e}}_{i j}^{*}=0, \forall i=1, \ldots, M, j \neq i,
\end{aligned}
$$

where

$$
\begin{aligned}
\mathbf{H}_{i k} & =\left(\mathbf{I}_{N} \otimes \mathbf{h}_{i k}\right)^{*}, \\
\mathbf{H} & =\sum_{k=1}^{M} \mathbf{H}_{1 k} \mathbf{H}_{1 k}^{H} \oplus \ldots \oplus \sum_{k=1}^{M} \mathbf{H}_{L k} \mathbf{H}_{L k}^{H}, \\
\overline{\mathbf{G}}_{i k} & =\left(\mathbf{I}_{N} \otimes \overline{\mathbf{g}}_{i k}\right)^{*}, \\
\overline{\mathbf{G}} & =\sum_{k=1}^{M} \overline{\mathbf{G}}_{1 k} \overline{\mathbf{G}}_{1 k}^{H} \oplus \ldots \oplus \sum_{k=1}^{M} \overline{\mathbf{G}}_{L k} \overline{\mathbf{G}}_{L k}^{H}, \\
\mathbf{d}_{i} & =\mathbf{Q}\left(\mathbf{I} \otimes \mathbf{u}_{i}^{*}\right) \underline{\mathbf{g}}_{i}, \\
\widetilde{\mathbf{d}}_{i} & =\mathbf{Q}\left(\mathbf{I} \otimes \widetilde{\mathbf{u}}_{i}^{*}\right) \underline{\mathbf{h}}_{i}, \\
\mathbf{e}_{i j} & =\mathbf{Q}\left(\mathbf{I} \otimes \mathbf{u}_{i}^{*}\right) \overline{\mathbf{g}}_{j}, \\
\widetilde{\mathbf{e}}_{i j} & =\mathbf{Q}\left(\mathbf{I} \otimes \widetilde{\mathbf{u}}_{i}^{*}\right) \underline{\mathbf{h}}_{j},
\end{aligned}
$$

and $\mathbf{u}_{i}, \widetilde{\mathbf{u}}_{i}, \overline{\mathbf{g}}_{i}, \underline{\mathbf{h}}_{i}$ and $\mathbf{Q}$ are defined in the following proof.

Proof. With the ZF constraint, (6) and (7) can be simplified as

$$
\mathrm{SNR}_{1}=\frac{P_{2} M}{\operatorname{tr}\left(\mathbf{D}_{1}\left(\sigma_{v}^{2} \sum_{i=1}^{L} \mathbf{H}_{i}^{T} \mathbf{W}_{i} \sum_{i=1}^{L} \mathbf{W}_{i}^{H} \mathbf{H}_{i}^{*}+\sigma_{x}^{2} \mathbf{I}\right) \mathbf{D}_{1}^{H}\right)}
$$

$$
\mathrm{SNR}_{2}=\frac{P_{1} M}{\operatorname{tr}\left(\mathbf{D}_{2}\left(\sigma_{v}^{2} \sum_{i=1}^{L} \mathbf{G}_{i} \mathbf{W}_{i} \sum_{i=1}^{L} \mathbf{W}_{i}^{H} \mathbf{G}_{i}^{H}+\sigma_{y}^{2} \mathbf{I}\right) \mathbf{D}_{2}^{H}\right)}
$$


From the property of $\operatorname{tr}($.$) , we may relax the inequality$ of $\mathrm{SNR}_{1}$ as

$$
\begin{aligned}
\mathrm{SNR}_{1} & =\frac{P_{2} M}{\operatorname{tr}\left(\mathbf{D}_{1} \mathbf{B D}_{1}^{H}\right)} \\
& =\frac{P_{2} M}{\operatorname{tr}\left(\mathbf{B D}_{1}^{H} \mathbf{D}_{1}\right)} \\
& \geq \frac{P_{2} M}{\operatorname{tr}(\mathbf{B}) \operatorname{tr}\left(\mathbf{D}_{1}^{H} \mathbf{D}_{1}\right)},
\end{aligned}
$$

where $\mathbf{B}=\left(\sigma_{v}^{2} \sum_{i=1}^{L} \mathbf{H}_{i}^{T} \mathbf{W}_{i} \sum_{i=1}^{L} \mathbf{W}_{i}^{H} \mathbf{H}_{i}^{*}+\sigma_{x}^{2} \mathbf{I}\right)$

Substituting (18) into (21) yields

$$
\mathrm{SNR}_{1} \geq \frac{P_{2} M}{\operatorname{tr}(\mathbf{B}) \sum_{i=1}^{M} \frac{1}{\left|\phi_{i i}\right|^{2} \sigma_{i}}} .
$$

From (16) and the definition of $\underline{\overline{\mathbf{W}}}$, we have

$$
\begin{aligned}
\boldsymbol{\Phi} & =\mathbf{U}^{H} \underline{\overline{\mathbf{W}}}=\mathbf{U}^{H} \underline{\mathbf{W}} \underline{\mathbf{G}}^{T} \\
& =\left(\begin{array}{c}
\operatorname{vec}(\underline{\mathbf{W}})^{H}\left(\mathbf{I} \otimes \mathbf{u}_{1}^{*}\right) \\
\vdots \\
\operatorname{vec}(\underline{\mathbf{W}})^{H}\left(\mathbf{I} \otimes \mathbf{u}_{M}^{*}\right)
\end{array}\right) \underline{\mathbf{G}}^{T} .
\end{aligned}
$$

Therefore, the elements of $\boldsymbol{\Phi}$ can be represented by

$$
\phi_{i j}=\operatorname{vec}(\underline{\mathbf{W}})^{H}\left(\mathbf{I} \otimes \mathbf{u}_{i}^{*}\right) \underline{\mathbf{g}}_{j},
$$

where $\mathbf{u}_{i}$ denotes the $i$ th column of $\mathbf{U}$ and $\underline{\mathbf{g}}_{j}^{T}$ denotes the jth row of $\underline{\mathbf{G}}$.

Similarly, for $\mathrm{SNR}_{2}$, we have

$$
\mathrm{SNR}_{2} \geq \frac{P_{1} M}{\operatorname{tr}(\widetilde{\mathbf{B}}) \sum_{i=1}^{M} \frac{1}{\left|\hat{\phi}_{i i}\right|^{2} \widetilde{\sigma}_{i}}},
$$

where $\widetilde{\mathbf{B}}=\left(\sigma_{v}^{2} \sum_{i=1}^{L} \mathbf{G}_{i} \mathbf{W}_{i} \sum_{i=1}^{L} \mathbf{W}_{i}^{H} \mathbf{G}_{i}^{H}+\sigma_{y}^{2} \mathbf{I}\right)$, and $\widetilde{\phi}_{i j}$ can be expressed by

$$
\widetilde{\phi}_{i j}=\operatorname{vec}(\underline{\mathbf{W}})^{H}\left(\mathbf{I} \otimes \widetilde{\mathbf{u}}_{i}^{*}\right) \underline{\mathbf{h}}_{j},
$$

where $\widetilde{\mathbf{u}}_{i}$ denotes the $i$ th column of $\widetilde{\mathbf{U}}$ and $\underline{\mathbf{h}}_{j}$ denotes the $j$ th column of $\underline{\mathbf{H}}$. It is assumed that the eigendecomposition of $\mathbf{G}^{H} \mathbf{G}$ is $\widetilde{\mathbf{U}} \tilde{\boldsymbol{\Lambda}} \tilde{\mathbf{U}}^{H}$.

Similar to Lemma 1, the trace of $\mathbf{B}$ and $\widetilde{\mathbf{B}}$ can be expressed as

$$
\begin{aligned}
& \operatorname{tr}(\mathbf{B})=\sigma_{v}^{2} \mathbf{w}^{H} \mathbf{H} \mathbf{w}+M \sigma_{x}^{2}, \\
& \operatorname{tr}(\widetilde{\mathbf{B}})=\sigma_{v}^{2} \mathbf{w}^{H} \overline{\mathbf{G}} \mathbf{w}+M \sigma_{y}^{2},
\end{aligned}
$$

where the definitions of $\mathbf{H}$ and $\overline{\mathbf{G}}$ are given in Lemma 3 .
Substituting (24) and (27a) into (22) and (26), and (27b) into (25) yields

$$
\begin{aligned}
\mathrm{SNR}_{1} & \geq \frac{P_{2} M}{\left(\sigma_{v}^{2} \mathbf{w}^{H} \mathbf{H w}+M \sigma_{x}^{2}\right) \sum_{i=1}^{M} \frac{1}{\left|\operatorname{vec}(\underline{\mathbf{W}})^{H}\left(\mathbf{I} \otimes \mathbf{u}_{i}^{*}\right) \underline{\mathbf{g}}_{i}\right|^{2} \sigma_{i}}}, \\
\mathrm{SNR}_{2} & \geq \frac{P_{1} M}{\left(\sigma_{v}^{2} \mathbf{w}^{H} \overline{\mathbf{G}} \mathbf{w}+M \sigma_{y}^{2}\right) \sum_{i=1}^{M} \frac{1}{\left|\operatorname{vec}(\underline{\mathbf{W}})^{H}\left(\mathbf{I} \otimes \tilde{\mathbf{u}}_{i}^{*}\right) \underline{\mathbf{h}}_{i}\right|^{2} \widetilde{\sigma}_{i}}} .
\end{aligned}
$$

From (28), (10) can be relaxed as

$$
\begin{gathered}
\frac{P_{2} M}{\gamma_{1}} \geq \sum_{i=1}^{M} \frac{\sigma_{v}^{2} \mathbf{w}^{H} \mathbf{H w}+M \sigma_{x}^{2}}{\left|\operatorname{vec}(\underline{\mathbf{W}})^{H}\left(\mathbf{I} \otimes \mathbf{u}_{i}^{*}\right) \underline{\mathbf{g}}_{i}\right|^{2} \sigma_{i}}, \\
\frac{P_{1} M}{\gamma_{2}} \geq \sum_{i=1}^{M} \frac{\sigma_{v}^{2} \mathbf{w}^{H} \overline{\mathbf{G}} \mathbf{w}+M \sigma_{y}^{2}}{\left|\operatorname{vec}(\underline{\mathbf{W}})^{H}\left(\mathbf{I} \otimes \widetilde{\mathbf{u}}_{i}^{*}\right) \underline{\mathbf{h}}_{i}\right|^{2} \widetilde{\sigma}_{i}} .
\end{gathered}
$$

If every term on the right side of (29a) and (29b) is smaller than $\frac{P_{2}}{\gamma_{1}}$ and $\frac{P_{1}}{\gamma_{2}}$, respectively, i.e.,

$$
\frac{P_{2}}{\gamma_{1}} \geq \frac{\sigma_{v}^{2} \mathbf{w}^{H} \mathbf{H w}+M \sigma_{x}^{2}}{\left|\operatorname{vec}(\underline{\mathbf{W}})^{H}\left(\mathbf{I} \otimes \mathbf{u}_{i}^{*}\right) \underline{\mathbf{g}}_{i}\right|^{2} \sigma_{i}}, \forall i=1, \ldots, M,
$$

$\frac{P_{1}}{\gamma_{2}} \geq \frac{\sigma_{v}^{2} \mathbf{w}^{H} \overline{\mathbf{G}} \mathbf{w}+M \sigma_{y}^{2}}{\left|\operatorname{vec}(\underline{\mathbf{W}})^{H}\left(\mathbf{I} \otimes \widetilde{\mathbf{u}}_{i}^{*}\right) \underline{\mathbf{h}}_{i}\right|^{2} \widetilde{\sigma}_{i}}, \forall i=1, \ldots, M$,

(29) can be satisfied.

Because $\underline{\mathbf{W}}$ is block diagonal matrices, there are many zero elements in $\operatorname{vec}(\underline{\mathbf{W}})$, which do not contribute to the calculation of (30). Suppose $\mathbf{Q}$ is chosen such that

$$
\mathbf{w}=\mathbf{Q v e c}(\underline{\mathbf{W}})
$$

holds.

To derive (30), we have make assumption that $\mathbf{D}_{1}^{H} \mathbf{D}_{1}$ and $\mathbf{D}_{2}^{H} \mathbf{D}_{2}$ should be diagonal. From (17), we may achieve this by forcing $\Phi$ to be a diagonal matrix. Therefore, the following equations should be satisfied:

$$
\begin{aligned}
& \phi_{i j}=\operatorname{vec}(\underline{\mathbf{W}})^{H}\left(\mathbf{I} \otimes \mathbf{u}_{i}^{*}\right) \underline{\mathbf{g}}_{j}=0, \forall i \neq j, \\
& \widetilde{\phi}_{i j}=\operatorname{vec}(\underline{\mathbf{W}})^{H}\left(\mathbf{I} \otimes \widetilde{\mathbf{u}}_{i}^{*}\right) \underline{\mathbf{h}}_{j}=0, \forall i \neq j .
\end{aligned}
$$

With (30) to (33) and definitions given in Lemma 3, (20) can be derived.

\section{Optimization of the proposed relay network}

In this section, we introduce optimization of the proposed two-way MIMO relay network using the following three criteria. 
Minimizing the total relay transmission power subject to individual minimal output SNR constraint and ZF constraint Using this criterion, the optimization problem is formulated as

$$
\min _{\mathbf{w}} P_{\mathrm{r}}
$$

subject to $\left(\mathrm{SNR}_{1}\right)_{\text {lower }} \geq \gamma_{1},\left(\mathrm{SNR}_{2}\right)_{\text {lower }} \geq \gamma_{2}$.

where $\left(\mathrm{SNR}_{1}\right)_{\text {lower }}$ and $\left(\mathrm{SNR}_{2}\right)_{\text {lower }}$ denote the minimal output SNR at transceiver 1 and transceiver 2, respectively.

Theorem 1. (34) can be approximated as an SOCP problem given as

$$
\begin{aligned}
& \min _{\mathbf{w}}\left\|\mathbf{U}_{1} \mathbf{w}\right\|_{2} \text {, } \\
& \text { subject to } \\
& \left\|\mathbf{U}_{2} \mathbf{w}^{\prime}\right\|_{2} \leq \frac{\operatorname{Real}\left\{\mathbf{w}^{H} \mathbf{d}_{i}\right\}}{\sqrt{\gamma_{1}}}, \forall i=1, \ldots, M \\
& \left\|\mathbf{U}_{3} \mathbf{w}^{\prime}\right\|_{2} \leq \frac{\operatorname{Real}\left\{\mathbf{w}^{H} \tilde{\mathbf{d}}_{i}\right\}}{\sqrt{\gamma_{2}}}, \forall i=1, \ldots, M, \\
& \operatorname{Imag}\left\{\mathbf{w}^{H} \mathbf{d}_{i}\right\}=0, \forall i=1, \ldots, M \text {, } \\
& \operatorname{Imag}\left\{\mathbf{w}^{H} \tilde{\mathbf{d}}_{i}\right\}=0, \forall i=1, \ldots, M \text {, } \\
& \mathbf{w}^{H} \mathbf{e}_{i j}^{*}=0, \forall i=1, \ldots, M, j \neq i \text {, } \\
& \mathbf{w}^{H} \widetilde{\mathbf{e}}_{i j}^{*}=0, \forall i=1, \ldots, M, j \neq i, \\
& \mathbf{w}^{\prime}=\left(\begin{array}{c}
\mathbf{w} \\
1
\end{array}\right) \text {. }
\end{aligned}
$$

and $\mathbf{d}_{i}, \widetilde{\mathbf{d}}_{i}, \mathbf{e}_{i j}$, and $\widetilde{\mathbf{e}}_{i j}$ are given in Lemma 3.

Proof. Define $\mathbf{A}_{i}=\left(\begin{array}{c}\overline{\mathbf{H}}_{i 1}^{H} \\ \vdots \\ \overline{\mathbf{H}}_{i M}^{H}\end{array}\right), \mathbf{B}_{i}=\left(\begin{array}{c}\mathbf{G}_{i 1}^{H} \\ \vdots \\ \mathbf{G}_{i M}^{H}\end{array}\right)$ and $\mathbf{U}_{1}=\left(\begin{array}{c}\sqrt{P_{1}}\left(\mathbf{A}_{1} \oplus \ldots \oplus \mathbf{A}_{L}\right) \\ \sqrt{P_{2}}\left(\mathbf{B}_{1} \oplus \ldots \oplus \mathbf{B}_{L}\right) \\ \sigma_{\nu} \mathbf{I}_{N L}\end{array}\right)$. From Lemma 1, P P can represented as $\left\|\mathbf{U}_{1} \mathbf{w}\right\|_{2}^{2}$. If we define $\mathbf{C}_{i}=\left(\begin{array}{c}\mathbf{H}_{i 1}^{H} \\ \vdots \\ \mathbf{H}_{i M}^{H}\end{array}\right)$ and $\mathbf{U}_{2}=\left(\begin{array}{cc}\sigma_{v}\left(\mathbf{C}_{1} \oplus \ldots \mathbf{C}_{L}\right) & \mathbf{0} \\ \mathbf{0}^{T} & \sqrt{M} \sigma_{x}\end{array}\right)$, the nominator of the left side of (20a) can be represented as $\left\|\mathbf{U}_{2} \mathbf{w}^{\prime}\right\|_{2}^{2}$. Similarly, we define $\mathbf{E}_{i}=\left(\begin{array}{c}\overline{\mathbf{G}}_{i 1}^{H} \\ \vdots \\ \overline{\mathbf{G}}_{i M}^{H}\end{array}\right)$ and $\mathbf{U}_{3}=$ $\left(\begin{array}{cc}\sigma_{\nu}\left(\mathbf{E}_{1} \oplus \ldots \mathbf{E}_{L}\right) & \mathbf{0} \\ \mathbf{0}^{T} & \sqrt{M} \sigma_{y}\end{array}\right)$, the nominator of the left side of (20b) can be represented as $\left\|\mathbf{U}_{3} \mathbf{w}^{\prime}\right\|_{2}^{2}$. Using these definitions, (20a) and (20b) can be expressed as

$$
\begin{aligned}
\left\|\mathbf{U}_{2} \mathbf{w}^{\prime}\right\|_{2}^{2} & \leq \frac{\mathbf{w}^{H} \mathbf{d}_{i} \mathbf{d}_{i}^{H} \mathbf{w}}{\gamma_{1}}, \forall i=1, \ldots, M, \\
\left\|\mathbf{U}_{3} \mathbf{w}^{\prime}\right\|_{2}^{2} & \leq \frac{\mathbf{w}^{H} \widetilde{\mathbf{d}}_{i} \widetilde{\mathbf{d}}_{i}^{H} \mathbf{w}}{\gamma_{2}}, \forall i=1, \ldots, M .
\end{aligned}
$$

From the fact that $\operatorname{Real}\{x\} \leq|x|$, (36) can be relaxed by

$$
\begin{aligned}
& \left\|\mathbf{U}_{2} \mathbf{w}^{\prime}\right\|_{2} \leq \frac{\operatorname{Real}\left\{\mathbf{w}^{H} \mathbf{d}_{i}\right\}}{\sqrt{\gamma_{1}}}, \forall i=1, \ldots, M, \\
& \left\|\mathbf{U}_{3} \mathbf{w}^{\prime}\right\|_{2}^{2} \leq \frac{\operatorname{Real}\left\{\mathbf{w}^{H} \widetilde{\mathbf{d}}_{i}\right\}}{\sqrt{\gamma_{2}}}, \forall i=1, \ldots, M, \\
& \operatorname{Imag}\left\{\mathbf{w}^{H} \mathbf{d}_{i}\right\}=0, \forall i=1, \ldots, M, \\
& \operatorname{Imag}\left\{\mathbf{w}^{H} \widetilde{\mathbf{d}}_{i}\right\}=0, \forall i=1, \ldots, M .
\end{aligned}
$$

Then, with Lemma 3, (35) can be derived.

\section{Maximizing the minimal output SNR of transceivers subject to total relay transmission power constraint and ZF constraint}

Assuming that the minimum SNR required by the two transceivers is $t$, the optimization problem can be formulated as

$\max _{\mathbf{w}} t$,

subject to $\left(\mathrm{SNR}_{1}\right)_{\text {lower }} \geq t,\left(\mathrm{SNR}_{2}\right)_{\text {lower }} \geq t, P_{\mathrm{r}} \leq P$.

where $P$ denotes the maximal total relay transmission power.

Theorem 2. (38) can be approximated as an SOCP problem:

$$
\begin{aligned}
& \max _{\mathbf{w}} t, \\
& \text { subject to }\left\|\mathbf{U}_{1} \mathbf{w}\right\|_{2} \leq \sqrt{P}, \\
& \left\|\mathbf{U}_{2} \mathbf{w}^{\prime}\right\|_{2} \leq \frac{\operatorname{Real}\left\{\mathbf{w}^{H} \mathbf{d}_{i}\right\}}{\sqrt{t}}, \forall i=1, \ldots, M, \\
& \left\|\mathbf{U}_{3} \mathbf{w}^{\prime}\right\|_{2} \leq \frac{\operatorname{Real}\left\{\mathbf{w}^{H} \widetilde{\mathbf{d}}_{i}\right\}}{\sqrt{t}}, \forall i=1, \ldots, M, \\
& \operatorname{Imag}\left\{\mathbf{w}^{H} \mathbf{d}_{i}\right\}=0, \forall i=1, \ldots, M, \\
& \operatorname{Imag}\left\{\mathbf{w}^{H} \widetilde{\mathbf{d}}_{i}\right\}=0, \forall i=1, \ldots, M, \\
& \mathbf{w}^{H} \mathbf{e}_{i j}^{*}=0, \forall i=1, \ldots, M, j \neq i, \\
& \mathbf{w}^{H} \widetilde{\mathbf{e}}_{i j}^{*}=0, \forall i=1, \ldots, M, j \neq i, \\
& \mathbf{w}^{\prime}=\left(\begin{array}{c}
\mathbf{w} \\
1
\end{array}\right) .
\end{aligned}
$$


Proof. It can be easily obtained from Lemmas 1 to 3 and Theorem 1.

Because (39) is quasi-convex, for any given value of $t$, it becomes the following SOCP problem:

$$
\begin{aligned}
& \text { find } \mathbf{w} \text {, } \\
& \text { subject to (39b) to (39i). }
\end{aligned}
$$

The bisection search procedure can be applied to solve (40).

\section{Maximizing the minimal output SNR of transceivers} subject to individual relay transmission power constraint and ZF constraint

The optimization problem is given as

$$
\max _{\mathbf{w}} t
$$

$$
\text { subject to }\left(\mathrm{SNR}_{1}\right)_{\text {lower }} \geq t,\left(\mathrm{SNR}_{2}\right)_{\text {lower }} \geq t \text {, }
$$

$$
P_{r i} \leq P_{i}, i=1, . ., L \text {, }
$$

where $P_{i}$ denotes the maximal transmission power of the $i$ th relay, and $P_{r i}=P_{1} \operatorname{tr}\left(\mathbf{W}_{i} \mathbf{H}_{i} \mathbf{H}_{i}^{H} \mathbf{W}_{i}^{H}\right)+$ $P_{2} \operatorname{tr}\left(\mathbf{W}_{i} \mathbf{G}_{i}^{T} \mathbf{G}_{i}^{*} \mathbf{W}_{i}^{H}\right)+\sigma_{v}^{2} \operatorname{tr}\left(\mathbf{W}_{i} \mathbf{W}_{i}^{H}\right)$.

Theorem 3. (41) can be approximated as an SOCP problem:

$$
\begin{aligned}
& \max _{\mathbf{w}} t, \\
& \text { subject to }\left\|\mathbf{U}_{1}^{i} \mathbf{w}\right\|_{2} \leq \sqrt{P_{i}}, i=1, \ldots, L, \\
& \left\|\mathbf{U}_{2} \mathbf{w}^{\prime}\right\|_{2} \leq \frac{\operatorname{Real}\left\{\mathbf{w}^{H} \mathbf{d}_{i}\right\}}{\sqrt{t}}, \forall i=1, \ldots, M, \\
& \left\|\mathbf{U}_{3} \mathbf{w}^{\prime}\right\|_{2} \leq \frac{\operatorname{Real}\left\{\mathbf{w}^{H} \widetilde{\mathbf{d}}_{i}\right\}}{\sqrt{t}}, \forall i=1, \ldots, M, \\
& \operatorname{Imag}\left\{\mathbf{w}^{H} \mathbf{d}_{i}\right\}=0, \forall i=1, \ldots, M, \\
& \operatorname{Imag}\left\{\mathbf{w}^{H} \widetilde{\mathbf{d}}_{i}\right\}=0, \forall i=1, \ldots, M, \\
& \mathbf{w}^{H} \mathbf{e}_{i j}^{*}=0, \forall i=1, \ldots, M, j \neq i, \\
& \mathbf{w}^{H} \widetilde{\mathbf{e}}_{i j}^{*}=0, \forall i=1, \ldots, M, j \neq i, \\
& \mathbf{w}^{\prime}=\left(\begin{array}{c}
\mathbf{w} \\
1
\end{array}\right),
\end{aligned}
$$

where $\mathbf{U}_{1}^{i}$ is defined as $\mathbf{U}_{r i}=\left(\begin{array}{c}\sqrt{P_{1}} \mathbf{A}_{i} \\ \sqrt{P_{2}} \mathbf{B}_{i} \\ \sigma_{\nu} \mathbf{I}_{N}\end{array}\right)$.

Proof. It can be easily obtained from Lemmas 1 to 3 and Theorem 1.
For any given value of $t$, (42) reduces to the following SOCP probelm:

$$
\begin{aligned}
& \text { find } \mathbf{w} \text {, } \\
& \text { subject to (42b) to (42i). }
\end{aligned}
$$

Similar to the solution of (40), (43) is solved by the bisection search procedure.

\section{Computer simulations}

In order to verify the validity of the proposed algorithm, we devise the following simulation scenario. The number of antennas of transceiver 1 , transceiver 2 , and relays is assumed to be $M=N=3$, and the number of relays is $L=10$. The communication channel coefficients are modeled by complex Gaussian variables with zero mean and variance $\sigma_{h}^{2}$ and $\sigma_{g}^{2}$. The two transceivers transmit independent data streams from different antennas with $P_{1}=P_{2}=0 \mathrm{~dB}$. AGN on each antenna is assumed to be complex Gaussian variable with zero mean and unit variance, i.e., $\sigma_{x}^{2}=\sigma_{y}^{2}=\sigma_{v}^{2}=0 \mathrm{~dB}$. Sources are generated from a QPSK constellation. The values of SNR are computed from 100 independent trials for each plot. Furthermore, the power consumption to increase the minimal output $\mathrm{SNR}_{2}$ for $2 \mathrm{~dB}$ becomes smaller as the value of $\gamma_{1}$ increases, which means the derived minimal output SNR approaches the output SNR as the value of SNR increases. Therefore, less additional power consumption is needed to increase the same amount of output SNR. This phenomenon can also be demonstrated by Figure 2.

\section{Minimizing the total relay transmission power subject to} individual minimal output SNR constraint and ZF constraint We assume that $\sigma_{h}^{2}=\sigma_{g}^{2}=0 \mathrm{~dB}$. Figure 3 depicts the total relay transmission power $P_{\mathrm{r}}$ against the value of $\gamma_{1}$. It is observed that the required transmission power increases as the value of $\gamma_{1}$ increases. Also, for a given $\gamma_{1}$, the total relay transmission power increases with the increase of $\gamma_{2}$.

Figure 2 shows the cumulative distribution function (CDF) of the output SNR at transceiver 2 with different values of $\gamma_{2}$. In Figure 2, the value of $\gamma_{2}$ is assumed to vary from -6 to $6 \mathrm{~dB}$ with $2 \mathrm{~dB}$ stepsize. From the figure, we see that for a given $\gamma_{2}$, the output SNR at transceiver 2 does not change significantly with the variation of $\gamma_{1}$, and the output $\mathrm{SNR}_{2}$ is about 2 to $3 \mathrm{~dB}$ higher than the value of $\gamma_{2}$ with $90 \%$ probability. This is reasonable since the proposed optimization problem uses the minimal output SNR instead of the real output SNR. It can be seen that the difference between the real output $\mathrm{SNR}_{2}$ and $\gamma_{2}$ decreases as the value of $\gamma_{2}$ increases, which is in accordance with the phenomenon observed in Figure 3.

Figure 4 plots the CDF of output $\mathrm{SNR}_{1}$ with different values of $\gamma_{2}$. It is found that the average output $\mathrm{SNR}_{1}$ 


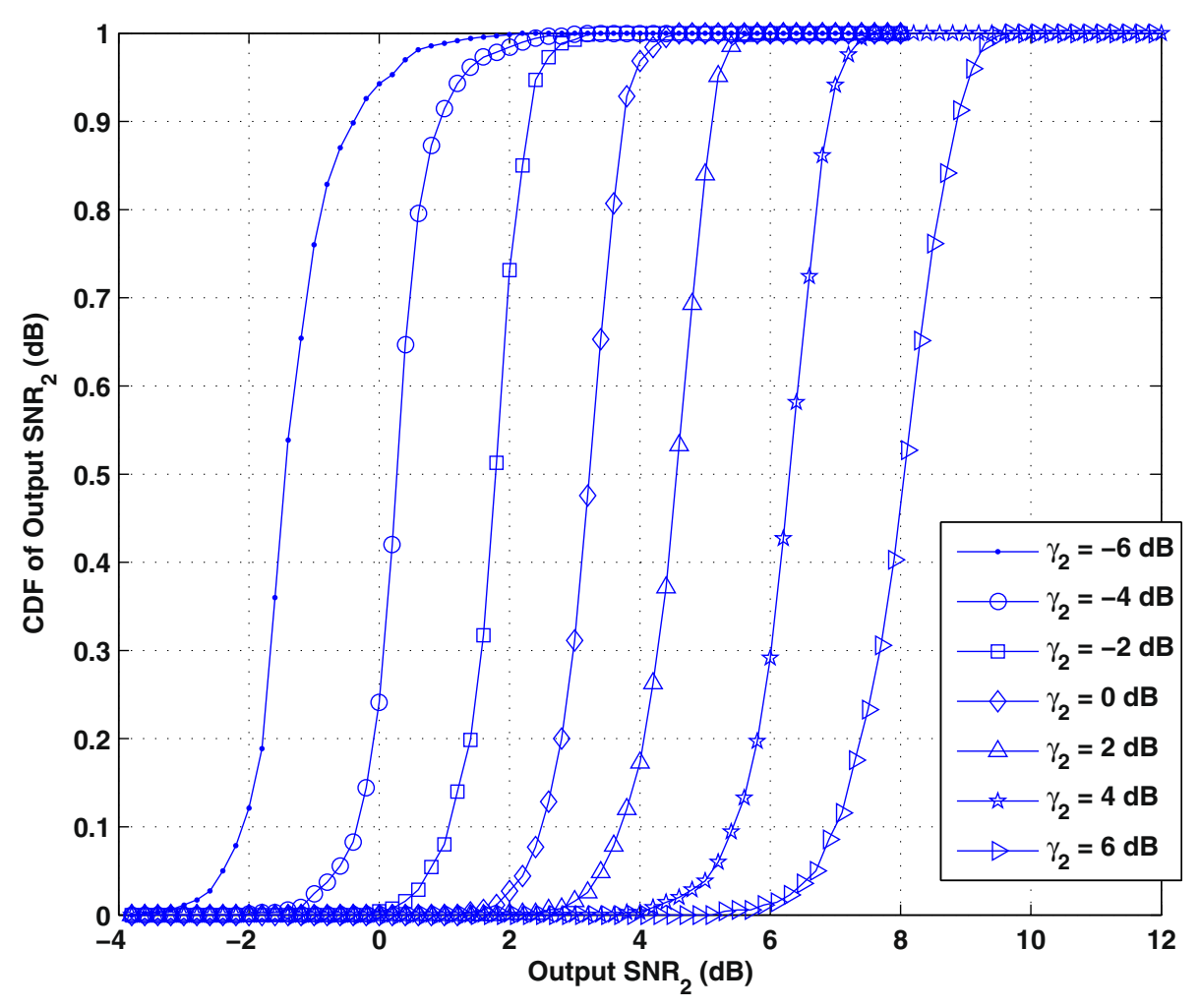

Figure 2 CDF of the output SNR at transceiver 2 with different values of $\gamma_{2}$.

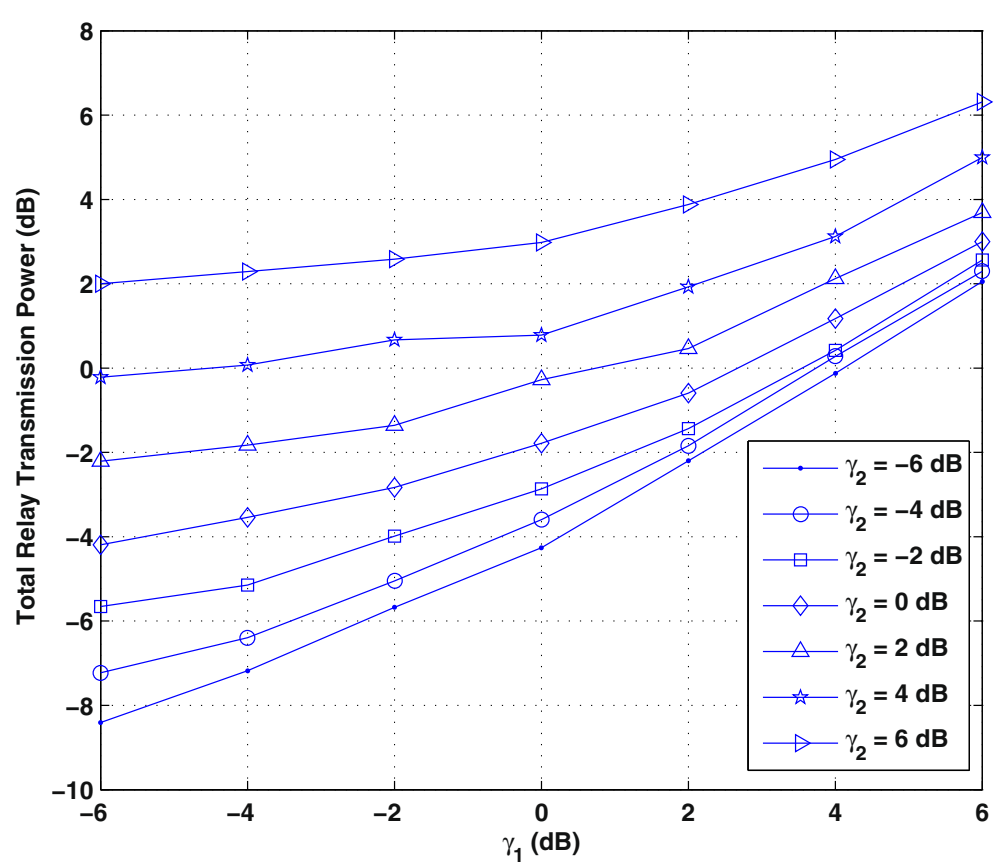

Figure 3 Total relay transmission power $P_{\mathrm{r}}$ versus the value of $\gamma_{1}$. 


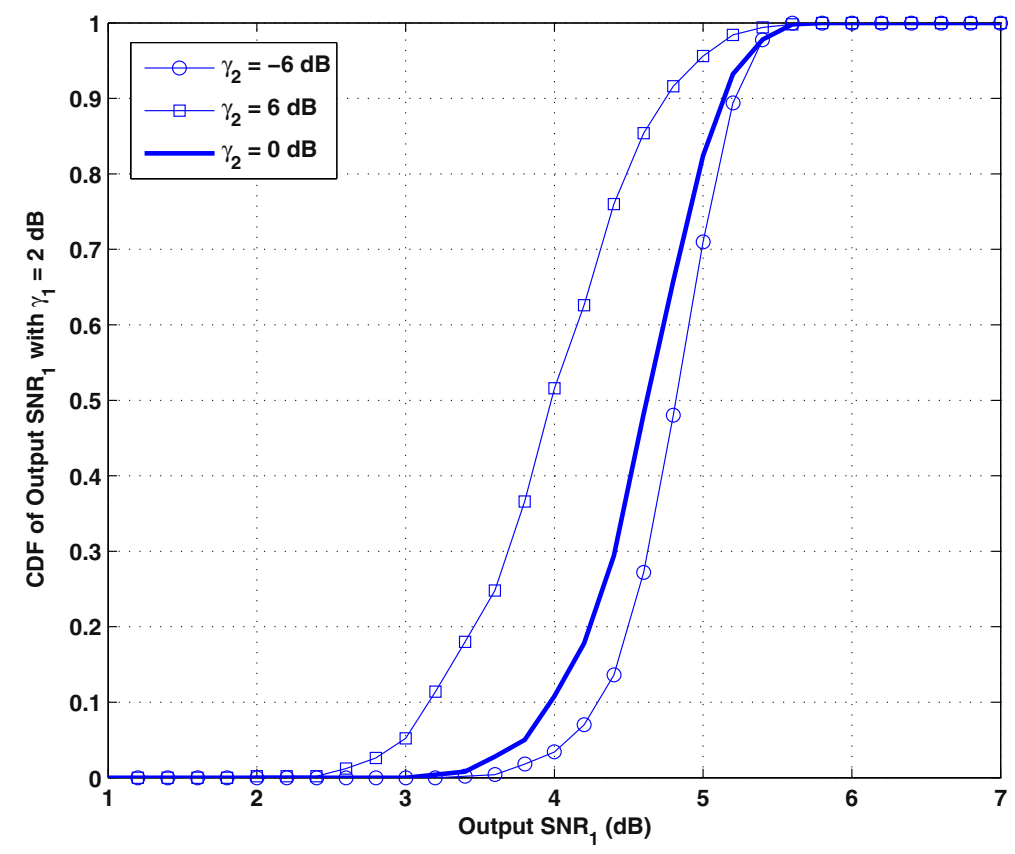

Figure $4 \mathrm{CDF}$ of the output SNR at transceiver 1 with different values of $\gamma_{2}$.

with $\gamma_{2}=6 \mathrm{~dB}$ is less than that with $\gamma_{2}=-6 \mathrm{~dB}$. It is known that $P_{\mathrm{r}}$ is allocated to the relays such that the two transceivers can simultaneously meet the required SNR. It can be concluded from Figure 4 that $P_{\mathrm{r}}$ allocation tends to emphasize maximizing the output SNR which has higher requirement under the condition that the lower $\mathrm{SNR}$ requirement can be satisfied. Therefore, $\mathrm{SNR}_{1}$ can achieve a higher average value when $\gamma_{2}=-6 \mathrm{~dB}$ than when $\gamma_{2}=6 \mathrm{~dB}$.

\section{Maximizing the minimal output SNR of transceivers subject to total relay transmission power constraint and ZF constraint}

Figure 5 depicts the output SNR at transceiver 1 with the value of $\sigma_{h}^{2}$ changing from 0 to $10 \mathrm{~dB}$. Total relay transmission powers of 0 and $5 \mathrm{~dB}$ are considered. It is found that for a given $\sigma_{h}^{2}$, the output $\mathrm{SNR}_{1}$ increases with the increase of $\sigma_{g}^{2}$, while for a given $\sigma_{g}^{2}, \mathrm{SNR}_{1}$ does not keep increasing with the increase of $\sigma_{h}^{2}$. This is because as the quality of channels between transceiver 1 and the relays improves, i.e., $\sigma_{h}^{2}$ increases, the desired transmission power at transceiver 2 to guarantee its output SNR increases [2]. Due to limitation of total relay transmission power, the output SNR at transceiver 1 can not increase consistently. When the quality of channels between transceiver 2 and the relays improves, the output $\mathrm{SNR}_{1}$ increases with the increase of $\sigma_{h}^{2}$.

Figure 6 shows the same plot for output $\mathrm{SNR}_{2}$. It is observed that the output $\mathrm{SNR}_{2}$ increases with the increase of $\sigma_{h}^{2}$, it while does not increase with the increase of $\sigma_{g}^{2}$ especially when $\sigma_{g}^{2}$ is high and $\sigma_{h}^{2}$ is relatively low. The reason is the same as that for $\mathrm{SNR}_{1}$ versus $\sigma_{h}^{2}$. Also, as noticed from Figures 5 and 6 , the output $\mathrm{SNR}_{1}$ and $\mathrm{SNR}_{2}$ increases with the increase of the total relay transmission power.

\section{Maximizing the minimal output SNR of transceivers subject to individual relay transmission power constraint and ZF constraint}

In this simulation, we assume that the total relay transmission power is uniformly allocated to the relays. Figures 7 and 8 show the output SNR versus the value of $\sigma_{h}^{2}$ with individual relay powers of -10 and $0 \mathrm{~dB}$. It is noted that these plots are similar to those with total relay transmission power constraint. With the increase of individual relay transmission power, output SNRs at transceiver 1 and transceiver 2 increase. Compared with Figures 5 and 6 , it is found that the output $\mathrm{SNR}_{1}$ and $\mathrm{SNR}_{2}$ are slightly lower with individual power constraint than those with total power constraint. This is because individual power constraint is more restrictive than the total power constraint.

\section{Conclusions}

In this paper, we focus on the optimization of a twoway MIMO relay network. The proposed optimization criteria yield three SOCP problems which can be solved 


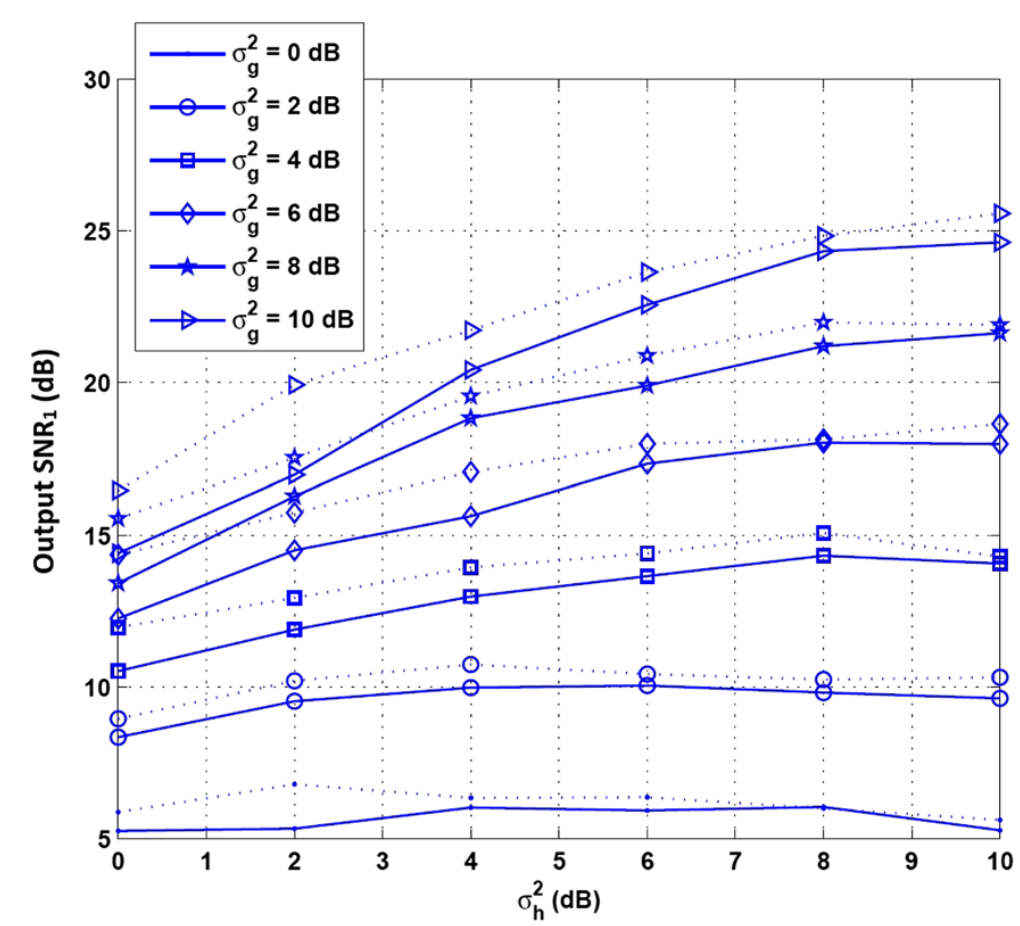

Figure 5 Output $\mathbf{S N R}_{\mathbf{1}}$ versus the value of $\boldsymbol{\sigma}_{\boldsymbol{h}}^{\mathbf{2}}$. Solid line: with total relay transmission power of $0 \mathrm{~dB}$, dash line: with total relay transmission power of $5 \mathrm{~dB}$.

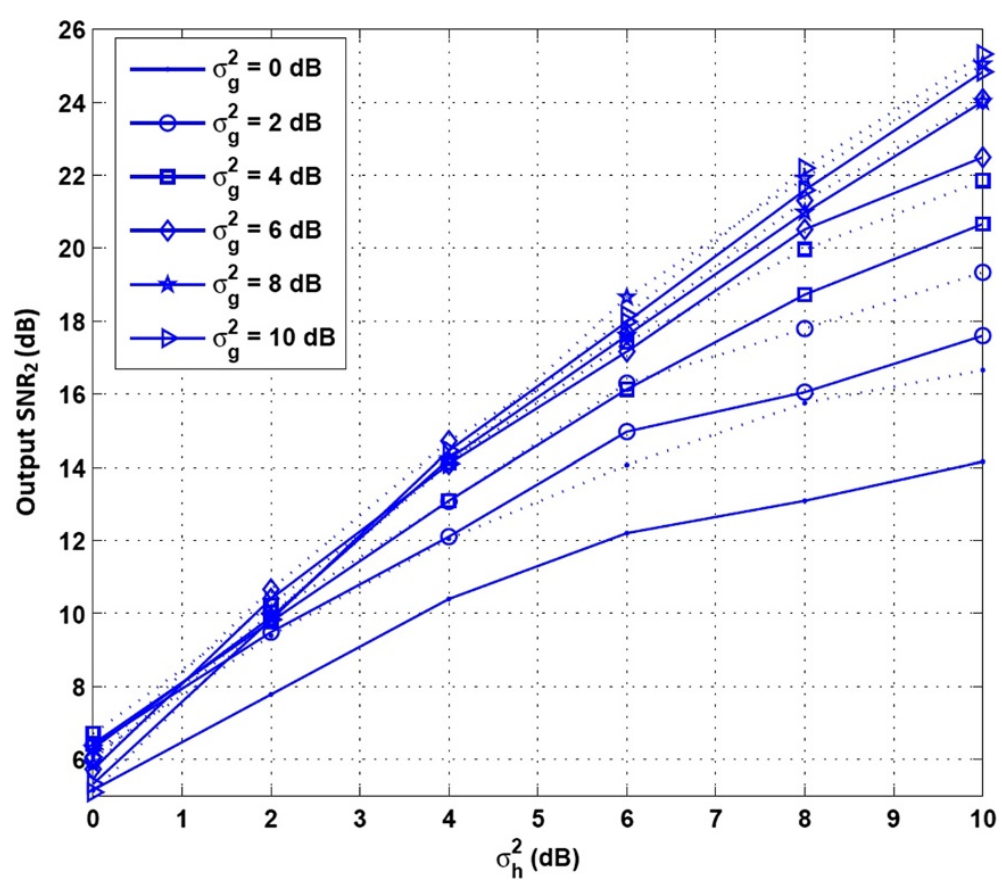

Figure 6 Output $\mathbf{S N R}_{\mathbf{2}}$ versus the value of $\boldsymbol{\sigma}_{\boldsymbol{h}}^{\mathbf{2}}$. Solid line: with total relay transmission power of $0 \mathrm{~dB}$, dash line: with total relay transmission power of $5 \mathrm{~dB}$ ). 


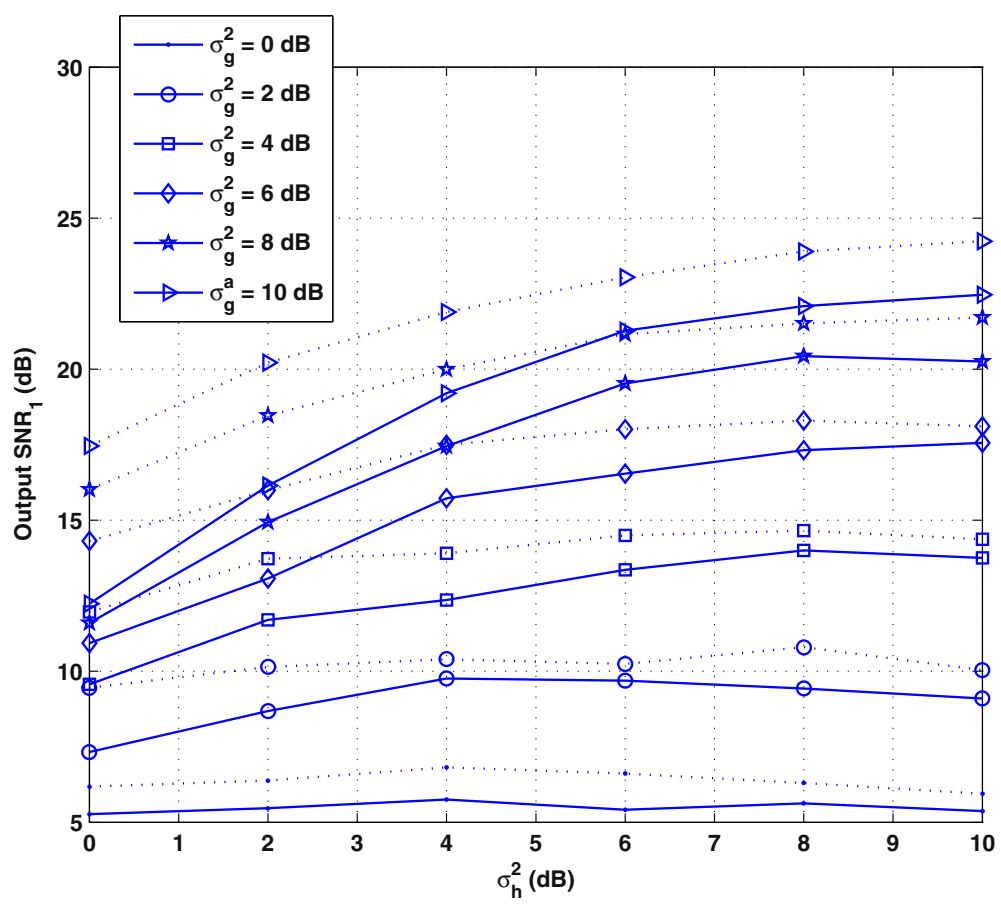

Figure 7 Output $\mathbf{S N R}_{\mathbf{1}}$ versus the value of $\boldsymbol{\sigma}_{\boldsymbol{h}}^{\mathbf{2}}$. Solid line: with individual relay transmission power of $-10 \mathrm{~dB}$, dash line: with individual relay transmission power of $0 \mathrm{~dB}$

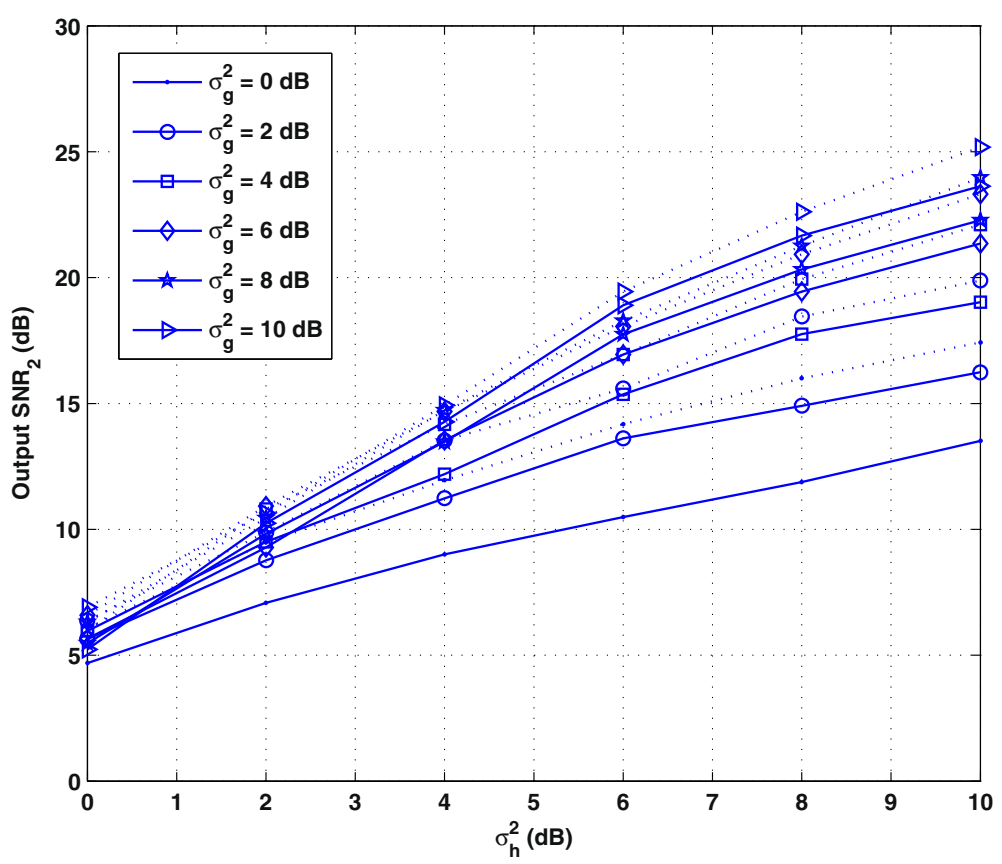

Figure 8 Output $\mathbf{S N R}_{2}$ versus the value of $\boldsymbol{\sigma}_{h}^{2}$. Solid line: with individual relay transmission power of $-10 \mathrm{~dB}$, dash line: with individual relay transmission power of $0 \mathrm{~dB}$ 
efficiently. Computer simulation demonstrates validity of the proposed algorithm. Furthermore, it is straightforward to see that the proposed algorithm can be implemented distributively as long as $\mathbf{U}$ and $\widetilde{\mathbf{U}}$ are broadcasted to all the relays. With $\mathbf{w}$ replaced by $\mathbf{w}_{i}, \mathbf{U}_{1}$ replaced by $\mathbf{U}_{r i}, \mathbf{U}_{2}$ replaced by $\left(\begin{array}{cc}\sigma_{\nu} \mathbf{C}_{i} & \mathbf{0} \\ \mathbf{0}^{T} & \sigma_{x}\end{array}\right)$, and $\mathbf{U}_{3}$ replaced by $\left(\begin{array}{cc}\sigma_{\nu} \mathbf{D}_{i} & \mathbf{0} \\ \mathbf{0}^{T} & \sigma_{y}\end{array}\right)$, (35), (39), and (42) can be solved at each relay. The performance of distributed implementation will be analyzed in our future work.

\section{Competing interests}

The authors declare that they have no competing interests.

\section{Acknowledgements}

The author wishes to acknowledge the financial support of the National Science Foundation of China through Grant No. 61101094 and No. 61201275.

\section{Author details}

${ }^{1}$ College of Electronic Engineering, University of Electronic Science and Technology of China, Xiyuan Avenue, 610066 Chengdu, China. ${ }^{2}$ Department of Electronics and Photonics, Institute of High Performance Computing, Fusionopolis Way, Singapore 138632, Singapore.

Received: 12 May 2014 Accepted: 8 December 2014

Published: 30 December 2014

\section{References}

1. G Zheng, Collaborative-relay beamforming with perfect CSI: optimum and distributed implementation. IEEE Signal Process Lett. 16(4), 257 (2009)

2. V Havary-Nassab, S Shahbazpanahi, A Grami, Z-Q Luo, Distributed beamforming for relay networks based on second-order statistics of the channel information. IEEE Trans. Signal Process. 56(9), 4306 (2008)

3. MM Abdallah, HC Pagadopoulos, Beamforming algorithms for implementation relaying in wireless sensor networks. IEEE Trans. Signal Process. 56(10), 4772 (2008)

4. E Koyuncu, $Y$ Jing, $H$ Jafarkhani, Distributed beamforming in wireless relay networks with quantized feedback. IEEE J. Selected Areas Commun. 26(8), 1429 (2008)

5. N Khajehnouri, AH Sayed, Distributed MMSE relay strategies for wireless sensor networks. IEEE Trans. Signal Process. 55(7), 3336 (2007)

6. H Chen, AB Gershman, S Shahbazpanahi, Filter-and-forward distributed beamforming in relay networks with frequency selective fading. IEEE Trans. Signal Process. 58(3), 1251 (2010)

7. V Havary-Nassab, S Shahbazpanahi, A Grami, Optimal distributed beamforming for two-way relay networks. IEEE Trans. Signal Process. 58(3), $1238(2010)$

8. H Chen, Beamforming Optimization for Two-Way Relay Channel (2014). p. 28-31

9. Y Rong, Optimal joint source and relay beamforming for MIMO relays with direct link. IEEE Commun. Lett. 14(5), 390 (2010)

10. B Khoshnevis, W Yu, R Adve, Grassmannian beamforming for MIMO amplify-and-forward relaying. IEEE J. Selected Areas Commun. 26(8), 1397 (2008)

11. W Guan, H Luo, Joint MMSE transceiver design in non-regenerative MIMO relay systems. IEEE Commun. Lett. J. 12(7), 517 (2008)

12. $\mathrm{Y}$ Rong, $\mathrm{F}$ Gao, Optimal beamforming for non-regenerative $\mathrm{MIMO}$ relays with direct link. IEEE Commun. Lett. J. 13(12), 927 (2009)

13. AS Behbahani, R Merched, AM Eltawil, Optimization of a MIMO relay network. IEEE Trans. Signal Process. 56(10), 5063 (2008)

14. K-J Lee, H Sung, E Park, I Lee, Joint optimization for one and two-way MIMO AF multiple-relay systems. IEEE Trans. Wireless Commun. 9(12), 3671 (2010)
15. A El-Keyi, B Champagne, Adaptive linearly constrained minimum variance beamforming for multiuser cooperative relaying using the Kalman filter. IEEE Trans. Wireless Commun. 9(2), 641 (2010)

16. J Joung, AH Sayed, Multiuser two-way amplify-and-forward relay processing and power control methods for beamforming systems. IEEE Trans. Signal Process. 58(3), 1833 (2010)

17. R Zhang, C Choy Chai, Y-C Liang, Joint beamforming and power control for multiantenna relay broadcase channel with QoS constraints. IEEE Trans. Signal Process. 57(2), 726 (2009)

18. O Oyman, AJ Paulray, Design and analysis of linear distributed MIMO relaying algorihms. IEEE Proc. Commun. 153(4), 565 (2006)

19. M Grant, S Boyd, cvx user's guide for cvx version 1.21 (build 790). http://cvxr.com/cvx/. Accessed 16 July 2013

doi:10.1186/1687-6180-2014-184

Cite this article as: Zhang et al:: Optimization of a two-way MIMO

amplify-and-forward relay network. EURASIP Journal on Advances in Signal Processing 2014 2014:184.

\section{Submit your manuscript to a SpringerOpen ${ }^{\mathcal{O}}$ journal and benefit from:}

$\checkmark$ Convenient online submission

Rigorous peer review

- Immediate publication on acceptance

Open access: articles freely available online

- High visibility within the field

- Retaining the copyright to your article

Submit your next manuscript at $\boldsymbol{\nabla}$ springeropen.com 